\title{
A clinical nomogram for the prediction of early mortality in elderly patients initiating dialysis for end-stage renal disease
}

\author{
Masaki Yoshida*, Masanori Otsuka, Yoshikazu Watanabe, Takako Harigai, Noriyuki Sakurai, Keiko Kobatake, \\ Hiroaki Yoshida, Satsuki Kobayashi, Takayuki Matsumoto, Tatsuhiko Sakamoto and Kazue Ueki
}

\begin{abstract}
Background: The number of elderly patients ( $>80$ years of age) with end-stage renal disease is rapidly increasing. The initiation of dialysis extends the duration of survival; however, the rate of early mortality, that which occurs within the first few months after the initiation of dialysis, is reportedly higher than the rate of late mortality.

Methods: We retrospectively studied a cohort of 300 patients, aged 80 years or older, in whom dialysis was initiated between January 1, 2010, and December 31, 2017, at TOHO Hospital (Gunma, Japan). The rate of early mortality was assessed using the Kaplan-Meier method, and the equivalence of survival curves was tested using log-rank tests. The univariate and multivariate analyses were performed using the Cox proportional hazards model. To evaluate nomogram performance, we assessed both the discrimination and calibration of these models. Two hundred bootstrap resamples were used for internal validation of the accuracy estimates to reduce overfit bias and to determine $95 \%$ confidence intervals.
\end{abstract}

Results: The nomogram was built using the following nine predictors: serum albumin grams per deciliter (hazard ratio [HR] 0.63, $p<0.001)$, congestive heart failure (HR 1.81, $p=0.004)$, chronic obstructive pulmonary disease (HR 2.47, $p=0.014$ ), peripheral vascular disease (HR 2.03, $p=0.019$ ), hemiplegia (HR 2.06, $p=0.001$ ), malignant tumors (no metastasis; HR 2.00, $p<0.001$ ), metastatic malignant tumors (HR 4.67, $p=0.006$ ), cardiovascular disease (HR 1.59, $p=0.002$ ), bone fractures due to falls within 1 year (HR 1.85, $p=0.011$ ), and Karnofsky Performance Status (HR 0.98, $p<0.001)$.

Conclusions: We developed and validated a nomogram that predicts early mortality in elderly patients at the initiation of dialysis for end-stage renal disease. The nomogram may help nephrologists make a shared decision with patients and families regarding the initiation of dialysis.

Keywords: Nomogram, Early mortality of dialysis initiation, End-stage renal disease, Elderly

\section{Background}

The demand for renal replacement therapy (RRT) is growing considerably in elderly people with end-stage renal disease (ESRD) in Japan, with the same needs trend all over the world. The number of Japanese dialysis patients who initiated dialysis at over 80 years of age was $25.1 \%$, a $10 \%$ increase in comparison with 10 years ago [1]. Generally, the initiation of dialysis extends the duration of survival; however, the rate of early mortality

\footnotetext{
* Correspondence: masakiyoshida-knz@umin.ac.jp

Dialysis and Nephrology Center, Sanshikai TOHO Hospital, 1155 Azami, Kasagake-machi, Midori, Gunma 379-2311, Japan
}

(mortality within the first few months after the initiation of dialysis) is reportedly higher than the rate of late mortality [2]. A recent analysis in Western countries found that the probability of early death within 3 months after initiation of dialysis ranged from 5.6 to $8.6 \%$, indicating that many patients do not survive long enough to benefit from dialysis [3]. Therefore, early death after initiating dialysis is a relevant problem, and the possibility of its occurrence influences medical decision-making. Nephrologists should recognize this problem before RRT and should discuss this with patients and families. Assessing the short-term prognosis is therefore important for

(c) The Author(s). 2020 Open Access This article is distributed under the terms of the Creative Commons Attribution 4.0 International License (http://creativecommons.org/licenses/by/4.0/), which permits unrestricted use, distribution, and 
individualizing care in patients with multiple comorbidities and functional limitations [4].

A prognostic nomogram would be a useful tool for evaluating RRT benefits and risks and informing patients and their families about treatment options.

\section{Materials and methods \\ Samples}

In this investigation, we included 300 patients aged 80 years and over, who started hemodialysis between 1 January 2009 and 31 December 2017 at our institution. The medical records were retrospectively reviewed, and overall survival rates were calculated by the KaplanMeier method.

\section{Patient characteristics}

Baseline information at dialysis initiation included age, sex, body mass index (BMI), the need for dialysis (as a scheduled or as an emergency procedure at initiation), comorbidities, laboratory data, Karnofsky Performance Status (KPS), and geriatric syndromes. Unplanned dialysis was defined as any first treatment begun under life-threatening circumstances requiring dialysis within $24 \mathrm{~h}$. The types of vascular access at dialysis initiation were catheter and arteriovenous fistula. Comorbidities were based on the Charlson comorbidity index. Comorbidities were as follows: diabetes mellitus, diabetes mellitus with end-organ damage (retinopathy, neuropathy, and nephropathy), congestive heart failure, cardiovascular disease (including a history of myocardial infarction, coronary vascular disease, coronary artery bypass, and angioplasty), collagen disease, peripheral vascular disease (history of gangrene or acute arterial insufficiency, or untreated thoracic or abdominal aneurysm $\geq 6 \mathrm{~cm}$ in size), cerebrovascular disease, chronic obstructive pulmonary disease (COPD), mild liver dysfunction (chronic hepatitis), moderate/severe liver dysfunction (cirrhosis and portal hypertension with variceal bleeding history), peptic ulcer (any history of treatment for ulcer disease or history of ulcer bleeding over the past year), no metastasis malignant tumor (excluded if 5 years from diagnosis and those found at the time of initiation and those currently being treated were included), metastatic malignant tumor, dementia, and hemiplegia. Laboratory data evaluated were serum albumin, sodium, potassium, chloride, phosphorus, corrected serum calcium, blood urea nitrogen, total cholesterol, hemoglobin, C-reactive protein, and estimated glomerular filtration rate. Geriatric syndromes included malnutrition (serum albumin and BMI), dementia, falls, and bone fracture (bone fracture from within 1 year of a fall).

\section{Statistical analysis}

Survival curves were made using the Kaplan-Meier method and compared using the log-rank test. All variables that achieved significance at $p$ values $<0.05$ in univariate analyses were enrolled in multivariate Cox proportional hazards model. The nomogram was formulated based on the results of multivariate analysis. A final model selection was performed using a backward stepdown selection process with the Akaike's information criterion (AIC). To evaluate nomogram performance, we assessed both the discrimination and calibration of these models. Two hundred bootstrap resamples were used for internal validation of the accuracy estimates, to reduce overfit bias and to determine 95\% confidence intervals (CI). This replicated the process of generating samples in the population, by drawing samples with replacements from the original dataset. The model, as estimated in the bootstrap sample, was evaluated in both the bootstrap sample and the original sample. The performance in the bootstrap sample represents an estimation of the apparent performance, and the performance in the original sample represents test performance. The difference between these performances is an estimation of the optimism in the apparent performance. This difference is averaged to obtain a stable estimate of the optimism. Bootstrapping offers the possibility of simulating the performance of the nomogram as if it were applied to future patients [5]. The analysis of timedependent area under receiver operating characteristic (ROC) curve was used to compare the discrimination power for 3-, 6-, and 12-month survival between the original and validation sets. The calibration plots of the original sets describe how far the predictions are from actual outcomes and how the prediction model fits the data by the Hosmer-Lemeshow test. The mean predicted 3-, 6-, and 12-month survival probabilities, averaged over the 200 models (validation sets), were compared with the average observed survival probabilities in the calibration plots. If the model is well calibrated, the plot should demonstrate agreement of the predicted and observed survival probabilities.

Statistical analyses were carried out using the SPSS24 $4^{\circ}$ for Windows and R software version 2.12.2.

\section{Ethics}

This study was performed with the approval of the Institutional Review Board of our institution.

\section{Results}

\section{Characteristics of the study population}

A total of 300 patients were analyzed. The mean age was $84( \pm 3.94)$ years old. The median follow-up period was 39.6 months (95\% CI 32.2-47.0). During this period, 147 patients $(49.0 \%)$ died. Survival for the original group of 
patients was estimated by Kaplan-Meier analysis (Fig. 1). The estimated median overall survival time was 39.6 months (95\% CI 32.2-47.0 months). The 3-, 6-, and 12month survival rates were $86.8 \%, 80.5 \%$, and $72.3 \%$ respectively. The patient characteristics are listed in Table 1.

\section{Independent prognostic factors in original data sets}

The data from the original sets were used to identify prognostic factors and build the model. The results of the univariate analysis are shown in Table 2. Variables considered significant in the univariate analyses were entered in the Cox multivariate analysis. A total of nine variables, serum albumin $(\mathrm{g} / \mathrm{dL})$ (hazard ratio [HR] 0.63, $p<0.001$ ), congestive heart failure (HR 1.81, $p=0.004$ ), chronic obstructive pulmonary disease (HR 2.47, $p=$ $0.014)$, peripheral vascular disease (HR 2.03, $p=0.019$ ), hemiplegia (HR 2.06, $p=0.001$ ), malignant tumor (no metastasis, HR 2.00, $p<0.001$ ), metastatic malignant tumor (HR 4.67, $p=0.006$ ), cardiovascular disease (HR $1.59, p=0.002)$, bone fracture due to fall within 1 year (HR 1.85, $p=0.011$ ), and Karnofsky Performance Status (HR 0.98, $p<0.001$ ), were proved independent in the multivariate Cox regression model and were incorporated in the nomogram according to the algorithm (Table 2).

\section{Prognostic nomogram}

Generally, each predictor is assigned a point range from 0 to 100 where the biggest impact predictor (such as a metastatic malignant tumor) is identified as a reference; the other predictors are then assigned based on their relative proportion to the biggest impact predictor. This is the principle of the point system in the nomogram.

Absolute maximum $\beta$ value $=\beta$ coefficient $\times$ value range of the predictor
For example, absolute maximum $\beta$ value of serum albumin is 0.453 ( $\beta$ coefficient) $\times 3.0$ (values of variable of serum albumin was 1.1 to 4.1 , and value range was 3.0 ) $=1.359$. The absolute maximum $\beta$ value of metastatic malignant tumor is 2.090 ( $\beta$ coefficient) $\times 1.0$ (values of variable of serum albumin was 0 to 1 , and value range was 1$)=2.090$, which means that it has the greatest impact on the probability of the event compared with the other predictors. The point system is constructed by firstly assigning 100 points to the metastatic malignant tumor, which has the greatest impact.

Once the point system of the predictor with the greatest impact is established, the remaining work is to assign other predictors in order, based on their proportion to the points assigned to the greatest impact predictor. For example, total point value of serum albumin is assigned based on this proportion to the total points given to metastatic malignant tumor.

Total point of serum albumin $=100 \times($ absolute $\max -$ imum $\beta$ value of serum albumin/absolute maximum $\beta$ value of metastatic malignant tumor $)=100 \times(1.359 /$ $2.090)=65.0$ points. The total points of malignant tumor $($ no metastasis) $=100 \times 0.691 / 2.09=33.0$ points Usually, positive $\beta$ coefficients are required in nomograms to simplify the calculation. We assigned 100 points to metastatic malignant tumor, 33 points to malignant tumor (no metastasis), and 0 points to no malignancy. The original $\beta$ value of serum albumin is negative; therefore, we assign 0 points to serum albumin $=4.1$ and 65 points to 1.1 reversely, thus avoiding subtractions when generating the total score (Table 3).

Using the data of patients in the original sets, a nomogram was developed to predict survival using the nine independent covariates identified in the multivariate model (Fig. 2). The nomogram is used by totaling the

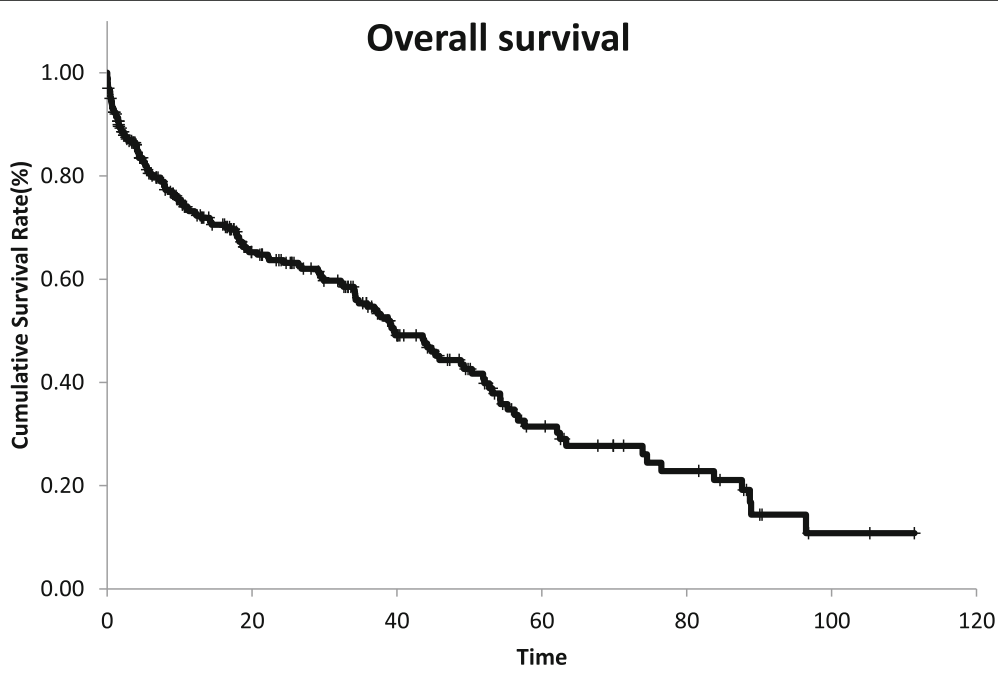

Fig. 1 Kaplan-Meier estimates for overall survival. The median survival time is 39.6 months (95\% Cl 32.2-47.0) 
Table 1 Patient characteristics

\begin{tabular}{|c|c|c|}
\hline \multicolumn{3}{|l|}{ Variables } \\
\hline Age (years) & Mean (SD) & $84(3.94)$ \\
\hline $80-84$ & $N(\%)$ & $191(63.7)$ \\
\hline $85-89$ & $N(\%)$ & $81(27)$ \\
\hline$>90$ & $N(\%)$ & $28(9.3)$ \\
\hline Sex (female) & $N(\%)$ & $139(46.3)$ \\
\hline Body mass index $\left(\mathrm{kg} / \mathrm{m}^{2}\right)$ & Mean (SD) & $19.6(3.4)$ \\
\hline$>22$ & $N(\%)$ & $93(31)$ \\
\hline $18.5-22$ & $N(\%)$ & $101(33.7)$ \\
\hline$<18.5$ & $N(\%)$ & $106(35.3)$ \\
\hline \multicolumn{3}{|l|}{ Comorbid conditions } \\
\hline Congestive heart failure & $N(\%)$ & $75(25)$ \\
\hline Cardiovascular disease & $N(\%)$ & $55(18.3)$ \\
\hline Peripheral vascular disease & $N(\%)$ & $10(3.3)$ \\
\hline Chronic obstructive pulmonary disease & $N(\%)$ & $15(5)$ \\
\hline Diabetes mellitus & $N(\%)$ & $123(41)$ \\
\hline Diabetes mellitus with end-organ damage & $N(\%)$ & $101(33.7)$ \\
\hline Cerebral infarction & $N(\%)$ & $79(26.3)$ \\
\hline Dementia & $N(\%)$ & $86(28.7)$ \\
\hline Collagen disease & $N(\%)$ & $15(5)$ \\
\hline Peptic ulcer & $N(\%)$ & $5(1.7)$ \\
\hline Hemiplegia & $N(\%)$ & $41(13.7)$ \\
\hline Mild liver dysfunction & $N(\%)$ & $10(3.3)$ \\
\hline Moderate/severe liver dysfunction & $N(\%)$ & $5(1.7)$ \\
\hline Malignant tumor & $N(\%)$ & $22(7.3)$ \\
\hline Metastatic malignant tumor & $N(\%)$ & $4(1.3)$ \\
\hline \multicolumn{3}{|l|}{ Laboratory data } \\
\hline Serum albumin g/dl & Mean (SD) & $2.8(0.6)$ \\
\hline Hemoglobin g/dl & Mean (SD) & $9.0(1.5)$ \\
\hline Serum sodium mEq/l & Mean (SD) & $137.6(5.2)$ \\
\hline Serum potassium mEq/l & Mean (SD) & $4.5(1.2)$ \\
\hline $\mathrm{eGFR} \mathrm{ml} / \mathrm{min} / 1.73 \mathrm{~m}^{2}$ & Mean (SD) & $8.5(3.9)$ \\
\hline Serum chloride mEq/l & Mean (SD) & $5.7(2.3)$ \\
\hline Corrected serum calcium mg/dl & Mean (SD) & $9.3(1.1)$ \\
\hline Blood urea nitrogen mg/dl & Mean (SD) & $81.3(36.2)$ \\
\hline Serum phosphorus mg/dl & Mean (SD) & $5.1(1.7)$ \\
\hline Total cholesterol mg/dl & Mean (SD) & $165.9(51.7)$ \\
\hline C-reactive protein $\mathrm{mg} / \mathrm{dl}$ & Mean (SD) & $2.9(5.5)$ \\
\hline \multicolumn{3}{|l|}{ Dialysis } \\
\hline Emergency initiation & $N(\%)$ & $116.0(38.7)$ \\
\hline Temporary catheter & $N(\%)$ & $144.0(48.0)$ \\
\hline Arteriovenous fistula & $N(\%)$ & $166.0(52.0)$ \\
\hline Karnofsky Performance Status & Mean (SD) & $60.1(23.8)$ \\
\hline $10-40$ & $N(\%)$ & $100.0(33.3)$ \\
\hline $50-70$ & $N(\%)$ & $97.0(32.7)$ \\
\hline $80-100$ & $N(\%)$ & $103.0(34.3)$ \\
\hline Fracture within 12 months & $N(\%)$ & $34.0(11.3)$ \\
\hline
\end{tabular}

points identified at the top scale for each independent covariate. This total point scale is used to identify the probability of 3-, 6-, and 12-month survival and to estimate median survival. For example, serum albumin 3.5 g/dL (13 points), peripheral vascular disease (33 points), and a Karnofsky Performance Status 40 (51 points) together have a total score of 97 points that is converted to a probability of survival rate. Three-, 6-, and 12month survival rates were $87.9 \%, 80.7 \%$, and $70.8 \%$, respectively.

\section{Internal validation of the nomogram (discrimination and calibration plots)}

In the original data sets, the concordance index (C-index) for overall survival (OS) was 0.827 (95\% CI 0.800-0.854; $p<0.001)$. The nomogram had good performance in early mortality prediction, with the time-dependent area under ROC curve (AUC) of 0.825 (95\% CI $0.76-0.89 ; p<0.001$ ), 0.816 (95\% CI $0.75-0.88 ; p<0.001)$, and 0.804 (95\% CI $0.75-0.87 ; p<0.001)$ at 3,6 , and 12 months, respectively. The C-index was calculated using the Cox proportional hazards model (Harrell's C-index) [5].

The prediction model was internally validated using bootstrap resampling, assessing its optimism-corrected discrimination and calibration [5, 6]. Across the 200 bootstrap repetitions, the median optimism-corrected Cindex for OS was 0.893 (95\% CI 0.84-0.94; $p<0.001$ ). Across the 200 bootstrap repetitions, the median optimism-corrected time-dependent AUC for 3-, 6-, and 12 -months survival was 0.828 (95\% CI $0.81-0.85 ; p<$ 0.001 ), 0.815 (95\% CI $0.80-0.83 ; p<0.001$ ), and 0.808 (95\% CI $0.79-0.83 ; p<0.001)$, respectively. Figure 3 shows a plot of the time-dependent optimism-corrected AUC indicating constant and adequate discriminative ability of our prediction model for prediction horizons between 1 and 60 months, especially within 12 months.

The bootstrap-predicted and the actual overall survival probabilities at 3-, 6-, and 12-months are shown in the calibration plots (Fig. 4a-c, respectively). The calibration plots describe how far the predictions are from the actual outcomes and how the prediction model fits the data by Hosmer-Lemeshow test $(p=0.619,0.582$, and 0.187 , respectively). This post hoc test illustrates whether the result of the calibration plot differs between the predicted value and the actual value.

\section{Discussion}

In our study, early mortality in elderly patients was high, even in those Japanese patients who had a better prognosis for dialysis.

Here, we developed and validated a novel prediction instrument for short-time mortality risk among elderly patients, using nine readily available baseline variables. The nine factors were classified into two groups, which 
Table 2 Cox proportional hazards model

\begin{tabular}{|c|c|c|c|c|c|c|c|c|c|c|}
\hline & \multicolumn{5}{|c|}{ Univariate Cox proportional hazard models } & \multicolumn{5}{|c|}{ Multivariate Cox proportional hazard models } \\
\hline & $\beta$ coefficient & $x^{2}$ & $p$ value & Hazard ratio & $95 \% \mathrm{Cl}$ & $\overline{\beta \text { coefficient }}$ & $x^{2}$ & $p$ value & Hazard ratio & $95 \% \mathrm{Cl}$ \\
\hline Age (year) & 0.014 & 0.405 & 0.525 & 1.015 & $0.970-1.061$ & & & & & \\
\hline Sex (female) & -0.132 & 0.628 & 0.428 & 0.876 & $0.632-1.214$ & & & & & \\
\hline Body mass index $\mathrm{kg} / \mathrm{m}^{2}$ & -0.020 & 0.560 & 0.454 & 0.981 & $0.932-1.032$ & & & & & \\
\hline Congestive heart failure & 0.374 & 4.213 & 0.040 & 1.454 & $1.017-2.078$ & 0.591 & 9.852 & 0.002 & 1.806 & $1.249-2.613$ \\
\hline Cardiovascular disease & 0.381 & 3.860 & 0.049 & 1.464 & $1.001-2.140$ & 0.467 & 5.406 & 0.020 & 1.594 & $1.076-2.363$ \\
\hline Peripheral vascular disease & 0.795 & 4.136 & 0.042 & 2.214 & $1.029-4.762$ & 0.706 & 2.911 & 0.005 & 2.026 & $0.900-4.558$ \\
\hline $\begin{array}{l}\text { Chronic obstructive } \\
\text { pulmonary disease }\end{array}$ & 0.878 & 6.334 & 0.012 & 2.406 & $1.214-4.767$ & 0.895 & 5.909 & 0.014 & 2.474 & $1.200-5.099$ \\
\hline Diabetes mellitus & 0.281 & 0.997 & 0.318 & 1.325 & $0.763-2.299$ & & & & & \\
\hline $\begin{array}{l}\text { Diabetes mellitus with } \\
\text { end-organ damage }\end{array}$ & -0.179 & 3.636 & 0.057 & 0.836 & $0.696-1.005$ & & & & & \\
\hline Cerebral infarction & 0.217 & 1.368 & 0.242 & 1.243 & $0.863-1.789$ & & & & & \\
\hline Dementia & 0.577 & 10.748 & 0.001 & 1.781 & $1.261-2.514$ & & & & & \\
\hline Collagen disease & 0.859 & 3.538 & 0.060 & 2.360 & $0.965-5.775$ & & & & & \\
\hline Peptic ulcer & 0.401 & 0.314 & 0.576 & 1.493 & $0.367-6.065$ & & & & & \\
\hline Hemiplegia & 0.378 & 12.052 & 0.001 & 1.460 & $1.179-1.808$ & 0.724 & 10.887 & 0.001 & 2.062 & $1.324-3.213$ \\
\hline Mild liver dysfunction & 0.137 & 0.090 & 0.764 & 1.147 & $0.468-2.809$ & & & & & \\
\hline $\begin{array}{l}\text { Moderate/severe liver } \\
\text { dysfunction }\end{array}$ & 0.068 & 0.159 & 0.690 & 1.070 & $0.768-1.491$ & & & & & \\
\hline $\begin{array}{l}\text { Malignant tumor } \\
\text { (no metastasis) }\end{array}$ & 0.605 & 20.945 & 0.000 & 1.831 & $1.413-2.372$ & 0.691 & 21.702 & 0.000 & 1.995 & $1.492-2.668$ \\
\hline $\begin{array}{l}\text { Metastatic malignant } \\
\text { tumor }\end{array}$ & 2.000 & 7.555 & 0.006 & 4.331 & $1.450-12.30$ & 2.090 & 7.555 & 0.006 & 4.668 & $1.556-14.00$ \\
\hline Serum albumin g/dl & -0.571 & 18.430 & 0.000 & 0.565 & $0.436-0.733$ & -0.453 & 9.793 & 0.002 & 0.634 & $0.477-0.843$ \\
\hline Hemoglobin g/dl & 0.021 & 0.132 & 0.716 & 1.021 & $0.913-1.143$ & & & & & \\
\hline Serum sodium mEq/l & -0.007 & 0.193 & 0.661 & 0.993 & $0.961-1.025$ & & & & & \\
\hline Serum potassium mEq/l & 0.068 & 0.801 & 0.371 & 1.070 & $0.923-1.241$ & & & & & \\
\hline eGFR ml/min/1.73 m² & 0.059 & 8.363 & 0.004 & 1.061 & $1.019-1.104$ & & & & & \\
\hline Serum chloride mEq/l & -0.024 & 4.148 & 0.042 & 0.977 & $0.955-0.999$ & & & & & \\
\hline $\begin{array}{l}\text { Corrected serum calcium } \\
\mathrm{mg} / \mathrm{dl}\end{array}$ & 0.387 & 12.290 & 0.000 & 1.473 & $1.186-1.829$ & & & & & \\
\hline Blood urea nitrogen mg/dl & 0.005 & 4.906 & 0.027 & 1.005 & $1.001-1.010$ & & & & & \\
\hline Serum phosphorus mg/dl & 0.089 & 2.477 & 0.115 & 1.093 & $0.978-1.221$ & & & & & \\
\hline Total cholesterol mg/dl & -0.003 & 2.783 & 0.095 & 0.997 & $0.994-1.001$ & & & & & \\
\hline C-reactive protein mg/dl & 0.060 & 29.335 & 0.000 & 1.062 & $1.039-1.085$ & & & & & \\
\hline Emergency initiation & 0.267 & 7.078 & 0.008 & 1.306 & $1.073-1.591$ & & & & & \\
\hline Initiation by catheter & 0.530 & 10.176 & 0.001 & 1.698 & $1.227-2.352$ & & & & & \\
\hline Karnofsky Performance Status & -0.023 & 38.373 & 0.000 & 0.977 & $0.970-0.984$ & -0.018 & 21.822 & 0.000 & 0.981 & $0.973-0.989$ \\
\hline Fracture within 12 months & 0.790 & 9.639 & 0.002 & 2.203 & $1.338-3.628$ & 0.617 & 6.440 & 0.011 & 1.853 & $1.111-3.089$ \\
\hline
\end{tabular}

included comorbidity factors and geriatric factors. The prediction nomogram reached sufficient accuracy and discriminated well.

Comorbidity factors were congestive heart failure, chronic obstructive pulmonary disease (COPD), peripheral vascular disease, hemiplegia, malignant tumor, and cardiovascular disease.

Patients with COPD had a 20\% increased risk of death compared with those patients who did not have COPD [7]. There were associations with various causes of death 
Table 3 Estimated coefficient and assigned points for each predictor

\begin{tabular}{|c|c|c|c|c|c|c|}
\hline & $\beta$ coefficient & Hazard ratio & Values of variable & Absolute maximum $\beta$ value & Rank & Assigned point \\
\hline Serum albumin $\mathrm{g} / \mathrm{dl}$ & -0.453 & 0.634 & 1.1 to 4.1 by 0.1 & 1.359 & 3 & 65 \\
\hline Congestive heart failure & 0.591 & 1.806 & 0.1 & 0.591 & 9 & 28 \\
\hline Chronic obstructive pulmonary disease & 0.895 & 2.474 & 0.1 & 0.895 & 4 & 43 \\
\hline Cardiovascular disease & 0.467 & 1.594 & 0.1 & 0.467 & 10 & 22 \\
\hline Peripheral vascular disease & 0.706 & 2.026 & 0.1 & 0.706 & 6 & 34 \\
\hline Hemiplegia & 0.724 & 2.062 & 0.1 & 0.724 & 5 & 35 \\
\hline Malignant tumor (no metastasis) & 0.691 & 1.995 & 0.1 & 0.691 & 7 & 33 \\
\hline Metastatic malignant tumor & 2.090 & 4.668 & 0.1 & 2.090 & 1 & 100 \\
\hline Karnofsky Performance Status & -0.018 & 0.981 & 100 to 20 by 10 & 1.440 & 2 & 69 \\
\hline Fracture within 12 months & 0.617 & 1.853 & 0.1 & 0.617 & 8 & 30 \\
\hline
\end{tabular}

in those with COPD and chronic kidney disease (CKD). The occurrence of COPD was associated with a fourfold higher risk of respiratory-related death in CKD [8]. It is noted that COPD is associated with an increased risk for sudden cardiac death. Whether such an increased risk for sudden cardiac death exists among those with COPD and CKD is unclear [8]. In this study, there were 15 patients with COPD (5.0\%), and 9 patients died, 3 patients died of respiratory death (pneumonia), and 2 patients died of cardiac death (myocardial infarction and heart failure).

Ten patients had peripheral vascular disease (3.3\%); of these, six patients died, two patients died of sepsis due to gangrene of the lower limbs, and one patient died of myocardial infarction and heart failure. For the overall Dialysis Outcomes and Practice Patterns Study (DOPPS) population, peripheral arterial disease (PAD) patients presented a significantly higher risk of all-cause death $(\mathrm{HR}=1.90)$, myocardial infarction-related death $(\mathrm{HR}=$ $1.48)$, and cardiac death $(H R=1.89)$ versus non-PAD patients in Japan [9]. Although the impact of PAD on survival is evident in all regions of all over the world, in Japan, patients both with and without PAD have a better survival rate than their counterparts in Europe and the USA/Canada [9]. In CKD stage V (pre-dialysis) patients, PAD is important in renal patients, not only as a marker of generalized cardiovascular disease, but also as a prognostic tool for higher mortality rates [10].

There were 22 patients with malignancy at the time of dialysis initiation $(7.3 \%)$, and 4 patients $(1.7 \%)$ were in a state of metastasis. Six patients $(27.2 \%)$ had urological cancer, and seven (31.8\%) had gastrointestinal cancer. Three patients were diagnosed by screening examinations at the time of admission for dialysis initiation. In the study by Masaki Hara et al., 9.6 to $32.3 \%$ patients were diagnosed with a new cancer at the time of dialysis initiation [11]. Cancer-related death was $47.1 \%$, and it was difficult to predict the convalescence of cancer at the time of dialysis initiation. A suggested approach to decision-making is summarized in the Renal Physicians Association (RPA) Shared Decision-Making in the Appropriate Initiation of and Withdrawal from Dialysis (2010) guidelines as a set of ten evidence-based recommendations that guide nephrologists through the dialysis decision-making process and provide indications for non-dialysis medical therapy and palliative care interventions [12].

Geriatric factors included malnutrition (serum albumin), bone fracture due to fall within 1 year, and Karnofsky Performance Status. These factors are consistent with other works that use surrogate markers for frailty. An index of malnutrition includes serum albumin, BMI, and weight loss. In this study of elderly patients, serum albumin was considered significant in the multivariate analysis, because both body weight and BMI increase due to body fluid retention at the time of dialysis initiation.

Activities of daily living (ADL) may be one of the most important prognostic factors, given the dramatic increase in the proportion of frail elderly patients on dialysis [13]. Functional status [severe (bedridden), moderate (overt difficulties in exerting basic ADL), or $\mathrm{mild} /$ none (none or some functional disabilities)] has been defined as the inability to perform ADL in the Japanese guideline for initiating dialysis [14]. A previous study reported that a severely to moderately impaired functional status was significantly associated with starting dialysis (HR 3.93 and 2.38, respectively) [15]. The RPA guidelines recommend considering foregoing dialysis for patients with CKD or ESRD who have a very poor prognosis or for whom dialysis cannot be provided safely. A significantly impaired functional status (Karnofsky Performance Status score $<40)$ is a very poor prognosis factor [16]. Impaired functional status can be caused by uremia and nonuremic factors such as aging, inflammation, and comorbidities (e.g., hemiplegia), as well as fractures from falls. 

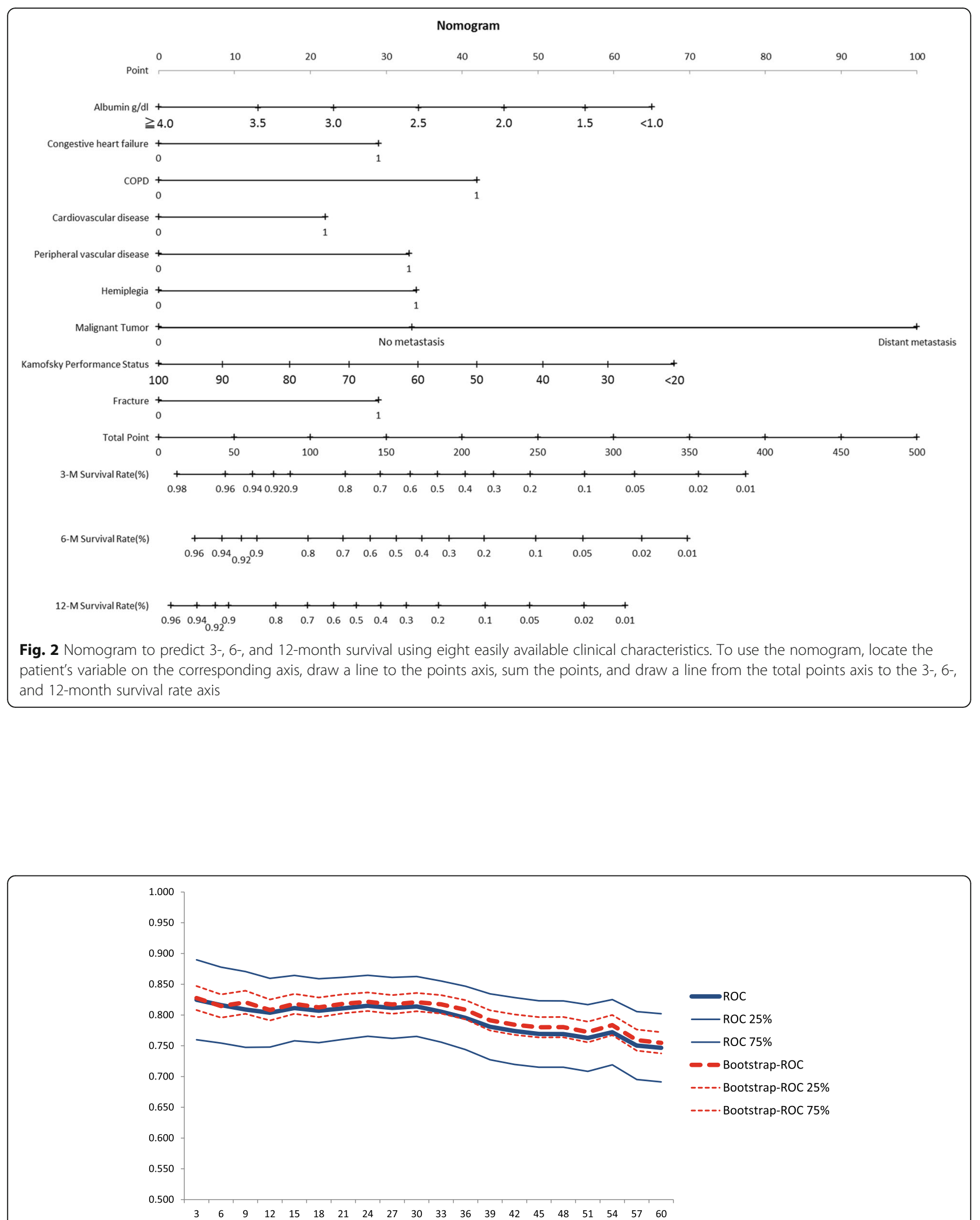

Fig. 3 Time-dependent discrimination curves. Optimism-corrected area under the ROC. Original set replicates shown as thick solid line and thin solid line denote 25th and 75th percentiles. Median over 200 bootstrap replicates shown as thick dashed line and thin dashed line denote 25th and 75 th percentiles 


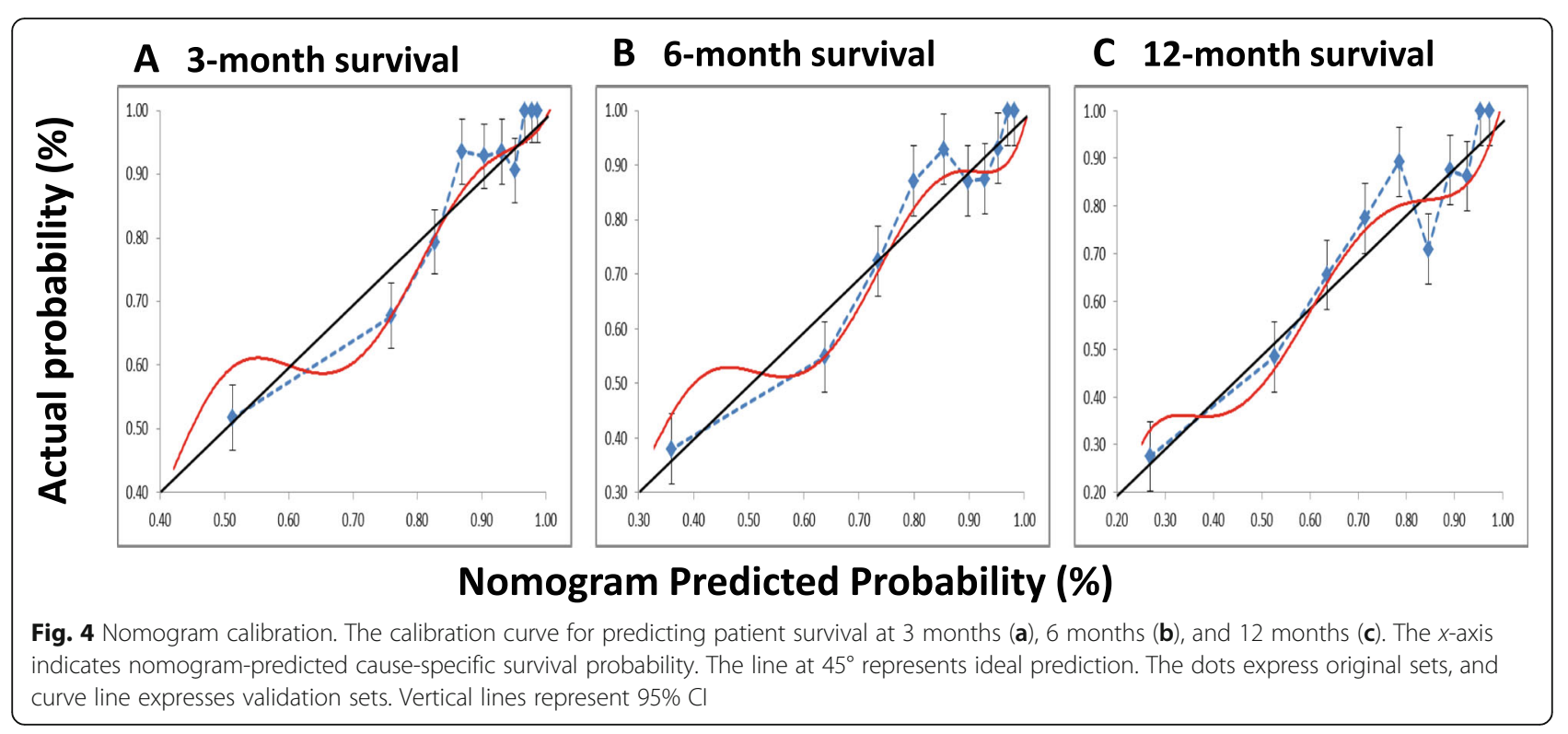

The prognostic predictors obtained in this study are consistent with those reported previously, and these factors are valid components of the prognostic nomogram.

In the elderly, multiple factors coexist, such as comorbidities and geriatric syndrome, and the importance of each factor varies. Therefore, the clinical use of nomograms, which provide accurate predictions from mathematical factors using multiple factors, is considered to be effective. It is important to use nomograms to make prognostic predictions that reflect the characteristics of each patient.

\section{Conclusion}

We developed and validated a nomogram that predicted early mortality in elderly patients starting dialysis for end-stage renal disease. Rather than doctors deciding on a medical treatment policy from a single point of view, multiple healthcare professionals, preferably the entire medical team, should examine and discuss the treatment policy. In addition, patients and their families often need explanation to help understand medical conditions and the merits and drawbacks of treatment. After careful consideration, the patient should be in agreement with the physicians and can choose an appropriate course of treatment (or no treatment). Importantly, the nomogram may help nephrologists make a shared decision with the patients and families regarding the initiation of dialysis, especially in elderly patients with many comorbidities. It is imperative to understand the potential risk presented by comorbidities and aging when starting dialysis in elderly patients. We are hopeful that this nomogram will become one of the indices used to evaluate such risks.

However, a major limitation of this study should be noted, in that it is necessary to perform external validation for estimating the accuracy of our nomogram, for it to be used in different institutions or for other patient populations.

\section{Acknowledgements \\ We are grateful to the members of the Dialysis and Nephrology Center, Sanshikai TOHO Hospital, for their important contributions to the treatment of patients.}

\section{Authors' contributions}

All authors collaborated in the data collection and analysis. As a dialysis and nephrology center administrator, KU contributed to the writing of the manuscript. All authors have read and approved the final manuscript.

\section{Funding}

The funding institution for the research design, data collection, analysis, interpretation, and manuscript writing was the Sanshikai Toho Hospital.

\section{Availability of data and materials}

The datasets analyzed during the current study are available from the corresponding author upon reasonable request.

Ethics approval and consent to participate

This study was performed with the approval of the Institutional Review Board of Sanshikai TOHO Hospital Ethics Committee (Clinical Research), reference number 18006.

\section{Consent for publication}

All patients have signed a comprehensive agreement to use the collected samples that accompany their treatment for future medical research. The contents of the research are thoroughly deliberated by the Ethics

Committee, and the data were used within the scope approved. If the patient refused or withdrew consent, there will be no penalty for medical treatment. The samples were anonymized to ensure that individuals could not be identified, and the utmost care was taken to protect personal privacy.

Competing interests

The authors declare that they have no competing interests. 
Received: 8 October 2019 Accepted: 4 February 2020

Published online: 13 February 2020

\section{References}

1. Masakane I, Nakai S et al. An overview of regular dialysis treatment in Japan (as of 31 2016). http://docs.jsdt.or.jp/overview/

2. Robinson B, Zhang J, Hal Morgenstern PD, et al. Worldwide, mortality risk is high soon after initiation of hemodialysis. Kidney Int. 2014 Jan;85(1):158-65. https://doi.org/10.1038/ki.2013.252 Epub 2013 Jun 26.

3. Noordzij M, Jager KJ. Increased mortality early after dialysis initiation: a universal phenomenon. Kidney Int. 2014;85(1):12-4

4. Koncicki HM, Swidler MA, et al. Decision making in elderly patients with advanced kidney disease. Clin Geriatr Med. 2013 Aug;29(3):641-55. https:// doi.org/10.1016/j.cger.2013.05.004.

5. Harrell FE Jr, Lee $\mathrm{KL}$, et al. Multivariable prognostic models: issues in developing models, evaluating assumptions and adequacy, and measuring and reducing errors. Stat Med. 1996;15:361.

6. Andrew J. Vickers Everything you always wanted to know about evaluating prediction models (but were too afraid to ask). Urology. 2010;76:1298-301.

7. Kent BD, Eltayeb EE, Woodman A, Mutwali A, Nguyen HT, Stack AG. The impact of chronic obstructive pulmonary disease and smoking on mortality and kidney transplantation in end-stage kidney disease. Am J Nephrol. 2012 36(3):287-95. https://doi.org/10.1159/000342207 Epub 2012 Sep 7.

8. Navaneethan SD, et al. Mortality outcomes of patients with chronic kidney disease and chronic obstructive pulmonary disease. Am J Nephrol. 2016:43:39-46.

9. Rajagopalan S, et al. Peripheral arterial disease in patients with end-stage renal disease observation from the Dialysis Outcomes and Practice Patterns Study (DOPPS). Circulation. 2006;114(18):1914-22. https://doi.org/10.1161/ CIRCULATIONAHA.105.607390.

10. Guerrero A, Montes $R$, et al. Peripheral arterial disease in patients with stages IV and V chronic renal failure. Nephrol Dial Transplant. 2006;21: 3525-31.

11. Hara M, Morita T, Nokiba $H$, et al. The mortality of cancer patients after the initiation of chronic hemodialysis therapy. J. Jpn. Soc.Dial.Ther. 2014;47(4): 235-40.

12. Rockville, MD et al. Renal physicians association. Shared decision-making in the appropriate initiation of and withdrawal from dialysis. 2nd ed. Renal physicans association. 2010

13. Canaud B, Tong L, et al. Clinical practices and outcomes in elderly hemodialysis patients: results from the Dialysis Outcomes and Practice Patterns Study (DOPPS). Clin J Am Soc Nephrol. 2011;6(7):1651-62.

14. Kawaguchi $Y$. National comparison: optimal peritoneal dialysis outcomes among Japanese patients. Perit Dial Int. 1999;19(suppl 3):S9-S16.

15. Masahiko Y, Ryo K, et al. Early mortality was highly and strongly associated with functional status in incident Japanese hemodialysis patients: a cohort study of the large national dialysis registry. PLOS ONE. 2016;11(6):e0156951.

16. Moss $\mathrm{AH}$, et al. Revised dialysis clinical practice guideline promotes more informed decision-making. Clin J Am Soc Nephrol. 2010;5:2380-3.

\section{Publisher's Note}

Springer Nature remains neutral with regard to jurisdictional claims in published maps and institutional affiliations.

Ready to submit your research? Choose BMC and benefit from:

- fast, convenient online submission

- thorough peer review by experienced researchers in your field

- rapid publication on acceptance

- support for research data, including large and complex data types

- gold Open Access which fosters wider collaboration and increased citations

- maximum visibility for your research: over $100 \mathrm{M}$ website views per year

At $\mathrm{BMC}$, research is always in progress.

Learn more biomedcentral.com/submissions 